masse, $e=$ Elektronenladung [negatives Vorzeichen], $\varphi=$ Austrittsarbeit der Elektronen).

Zur Berechnung von $B$ muß die Austrittsarbeit $\varphi$ der Elektronen vom Metall ins Vakuum bekannt sein, sonst gehen außer der Temperatur $T$ nur universelle Konstanten in Gl. (9) ein. $\varphi$ und $y$ wird für Gold und Platin wenig verschieden sein, nämlich $\varphi=4$ bis $6,5 \mathrm{eV}, y=3$ bis $6 \AA$. $B$ ist (wegen des negativen Vorzeichens von $e$ ) für beide Materialien dem Vorzeichen nach negativ und von der Größenordnung 1 bis $5 \cdot 10^{-6}\left[\mathrm{~V} \mathrm{Grad}^{-1}\right]$.

Die Thermokraft eines mit einer einmolekularen Fremdschicht bedeckten Goldkontaktes ergibt sich durch Addition einer Zahl der gleichen Größenordnung zum Thomson-Koeffizienten $\mu=+1,6$ $\cdot 10^{-6}\left[\mathrm{VGrad}^{-1}\right]$ (nach Borelius). Man berechnet

$$
{ }^{c} \mathrm{Au} \sim 3 \text { bis } 7 \cdot 10^{-6}\left[\mathrm{~V} \mathrm{Grad}^{-1}\right] .
$$

Experimentell wurde gefunden 0,6 bis $1,8 \cdot 10^{-6}$ $\left[\mathrm{VGrad}^{-1}\right]$. Bei Platin ist der Thomson-Koeffizient negativ und beträgt $\mu \sim-8 \cdot 10^{-6}\left[\mathrm{~V} \mathrm{Grad}^{-1}\right]$. Nach der Theorie erwartet man also für $c_{\mathrm{Pt}} \sim-3$ bis $-7 \cdot 10^{-6}\left[\mathrm{VGrad}^{-1}\right]$; gemessen wurde $\sim-2$ $\cdot 10^{-6}\left[\mathrm{~V} \mathrm{Grad}^{-1}\right]$.

Da die Ermittlung von $B$ nicht ganz frei von Willkür ist, und bei der experimentellen Bestimmung des Thomson-Koeffizienten wegen der Schwierigkeit der Messung und der starken Ab- hängigkeit von der Reinheit des Materials keine große Genauigkeit erreicht werden kann, darf die Ubereinstimmung als befriedigend angesehen werden. Vor allem ist es verständlich, daß sich die gleiche Größenordnung und das umgekehrte Vorzeichen für $c$ bei Gold und Platin ergibt, trotz der recht verschiedenen Größe von $|\mu|$.

Zum Schluß untersuchen wir, ob die S. 484 erwähnte Abnahme der Thermokraft bei dickeren Fremdschichten bei Platin ebenfalls aus theoretischen Überlegungen erklärt werden kann. Aus den Widerstandsmessungen bei Drähten im Anlieferungszustand war zu ersehen, daß die Schichten noch so dünn waren, daß der Tunneleffekt für die Stromleitung maßgebend war $(y<30 \AA)$. Gl. (6) bleibt daher näherungsweise anwendbar. Die Austrittsarbeit $\varphi$ wird nun mit zunehmender Schichtdicke kleiner, da bei dickeren Schichten nicht mehr mit der Austrittsarbeit Metall-Vakuum, sondern mit der Austrittsarbeit MetallFremdschicht (Halbleiter) zu rechnen ist ${ }^{6}$. Aus Gl. (9) ergibt sich, daß sowohl mit zunehmender Schichtdicke $y$ als auch mit abnehmender Austrittsarbeit $\varphi$ der Absolutwert von $B$ wächst, woraus folgt. daß bei Platin bei dickeren Schichten von $|\mu|$ ein größerer Wert abgezogen werden muß als bei einmolekularen Schichten. Die Thermokraft $c$ muß demnach kleiner werden, in Übereinstimmung mit dem Experiment.

\title{
Über sphärische elektromagnetische Eigenschwingungen in Räumen, die Plasmen enthalten
}

\author{
Von Winfried Otto Schumann \\ Aus dem Elektrophysikalischen Institut der Technischen Hochschule München \\ (Z. Naturforschg. 4 a, 486-491 [1949]; eingegangen am 3. April 1949)
}

\begin{abstract}
Es wird für eine mit Plasma gefüllte Hohlkugel, für eine dielektrische und eine leitende Kugel in einer Plasmaatmosphäre und für eine Plasmakugel in Luft gezeigt, inwieweit die frequenzabhängige Dielektrizitätskonstante die Schwingungsvorgänge ändert, und insbesondere inwieweit die Möglichkeit einer negativen Dielektrizitätskonstanten neue Schwingungstypen ergibt.
\end{abstract}

$\mathrm{F}$ ür eine Reihe von Problemen, die bei kurzen Wellen in Gasentladungen oder sonstwie ionisierten Medien auftreten, ist es erwünscht, zu wissen, wie sich durch das Vorhandensein eines Plasmas bisher schon bekannte hochfrequente Schwingungsfelder und Eigenfrequenzen ändern. Es soll daher im folgenden an einer Reihe besonders einfacher Schwingungstypen gezeigt wer- den, wie sie bei Anwesenheit eines Plasmas verlaufen, wobei insbesondere die geringsten möglichen Eigenfrequenzen gesucht sind. Das Plasma sei bestimmt durch seine Dielektrizitätskonstante (D.K.) $\varepsilon_{\mathrm{P}}=1-\left(\omega_{0}^{2} / \omega^{2}\right)$ und seine Eigenfrequenz $\omega_{0}{ }^{2}=\frac{N e^{2}}{\varepsilon_{0} m}$. Seine Dämpfung ist vernachlässigt. Die hochfrequenten Felder seien bestimmt durch ein 
Vektorpotential $\mathfrak{A}$, die Zeitabhängigkeit durch $\exp (j \omega t)$.

$\mathfrak{B}=\operatorname{rot} \mathfrak{A}$

oder

$\mathfrak{D}=\operatorname{rot} \mathfrak{A}$

$\mathfrak{E}=-j \omega \mathfrak{A}-\operatorname{grad} \varphi$, $\mathfrak{d}=j \omega \mathfrak{A}+\operatorname{grad} \mathscr{q}$, $\operatorname{rot} \operatorname{rot} \mathfrak{A}=\frac{\omega^{2}}{v^{2}} \mathfrak{A}-j j \frac{\omega}{v^{2}} \operatorname{grad} \varphi$,

$$
v^{2}=\frac{c^{2}}{\varepsilon ! "} \text {. }
$$

Wird $\mathfrak{A}$ in einem sphärischen Koordinatensystem $r, \Theta, \varphi$ parallel zum Radiusvektor gewählt, so ist im 1 . Fall $\mathfrak{B}$ senkrecht zum Radiusvektor (transversale $H$-Welle), im Falle 2 steht dann $\mathcal{I}$ senkrecht dazu (transversale $E$-Welle). Ist $A$ der Betrag von $\mathfrak{A}$, so ist mit

$$
-j \frac{\omega}{v^{2}} \varphi=\operatorname{div} \mathfrak{A}=\frac{\partial A}{\partial r},
$$

eine partikuläre Lösung

$$
\begin{gathered}
A=\exp (j m \varphi) P_{l}^{m}(\cos \Theta) \sqrt{r} Z_{l+1 / 2}(l r r), \\
k=\omega / v .
\end{gathered}
$$

$P_{l}^{m}$ ist die zugeordnete Kugelfunktion, $Z$ eine Bessel-Funktion. Wir beschränken uns auf den einfachen Fall $m=0, l=1$ und erhalten

$$
A=\sqrt{r} Z_{3 / 2}(k r) \cos \Theta .
$$

Dann wird für die transversale $H$-Welle

$$
\begin{gathered}
B_{\varphi}=\frac{k}{\sqrt{k r}} Z_{3 / 2}(k r) \sin \Theta, \\
E_{r}=-j \omega \frac{2}{(k r)^{3 / 2}} Z_{3 / 2}(k r) \cos \Theta,
\end{gathered}
$$

$E_{\Theta}=-j \omega \frac{1}{(k r)^{3 / 2}}\left[Z_{3 / 2}(k r)-k r Z_{1 / 2}(k r)\right] \sin \Theta$,

und für die transversale $E$-Welle

$$
\begin{gathered}
D_{\varphi}=\frac{k}{\sqrt{k r}} Z_{3 / 2}(k r) \sin \Theta \\
H_{r}=j \omega \frac{2}{(k r)^{3 / 2}} Z_{3 / 2}(k r) \cos \Theta \\
H_{\Theta}=j \omega \frac{1}{(k r)^{3 / 2}}\left[Z_{3 / 2}(k r)-k r Z_{1 / 2}(k r)\right] \sin \Theta .
\end{gathered}
$$

\section{Leitende Hohlkugel, mit Plasma gefüllt}

Es kommen nur Bessel-Funktionen 1. Art in Frage. a) Transversale $H$-Welle. An der Kugeloberfläche $r=a$ muß $E_{\Theta}=0$ sein.

Daraus folgt ${ }^{1} \operatorname{tg} k a=\frac{k a}{1-(k \cdot a)^{2}}$ mit der kleinsten Wurzel $k a=2,74$. Da $k^{2}=\omega^{2} / v^{2}=\left(1 / c^{2}\right)\left(\omega^{2}-\omega_{0}{ }^{2}\right)$, ist die Eigenfrequenz

$$
\omega_{\mathrm{ei}}^{2}=\omega_{\mathrm{v}}^{2}+\frac{7,51}{a^{2}} c^{2} .
$$

Der zweite Teil dieses Ausdruckes gibt die Eigenfrequenz für die mit Luft gefüllte Kugel, $\omega_{0}=0$, $\varepsilon_{r}=1$. Die Eigenfrequenz $\omega^{\omega}{ }_{\mathrm{ei}}$ ist also immer größer als die Eigenfrequenz $\omega_{0}$ des Plasmas. Der Unterschied beider hängt davon $a b$, ob $\omega_{0} \lessgtr$ $(2,74 / a) c$

b) Transversale $E-W e l l e$. In diesem Falle müssen für $r=a, D_{\varphi}$ und $H_{r}=0$ werden, woraus folgt $\operatorname{tg} k a=k a$, mit der kleinsten endlichen Wurzel $k a=4,49$.

Dann wird

$$
\omega_{\mathrm{ei}}^{2}=\omega_{0}^{2}+\left(4,49 c / a_{\rho^{2}}^{2} .\right.
$$

Also auch hier ist die Eigenfrequenz größer als $\omega_{0}$, und $\varepsilon_{\mathbf{P}}$ daher positiv.

$$
\begin{aligned}
& \text { II. Dielektrische Kugel } \\
& \text { in Plasmaatmosphäre }
\end{aligned}
$$

(Ungedämpfte Wellen, keine Strahlung)

In diesem Fall ist für den Innenraum (i) die Bessel-Funktion 1. Art und im Außenraum (Plasma, a) die Hankelsche Funktion einzuführen (2. Art, nach außen strahlend, mit negativ imaginärem Argument, d. h. $\varepsilon_{\mathrm{P}}<1, \omega<\omega_{0}$, wobei das Feld nach außen exponentiell abfällt).

a) Für die transversale $H$-Welle gilt wegen der Stetigkeit von $B_{\varphi}$ und $E_{\Theta}$ für $r=a$

$$
\begin{gathered}
\frac{1}{m_{\mathrm{i}}^{2}}\left[1-\frac{m_{\mathrm{i}}}{l} \frac{Z_{l-1 / 2}^{i}\left(m_{\mathrm{i}}\right)}{Z_{l+1 / 2}^{i}\left(m_{\mathrm{i}}\right)}\right] \\
=\frac{1}{m_{\mathrm{a}}^{2}}\left[1-\frac{m_{\mathrm{a}}}{l} \frac{Z_{l-1 / 2}^{n}\left(m_{\mathrm{a}}\right)}{Z_{l+1 \mid 2}^{i}\left(m_{\mathrm{a}}\right)}\right], \\
m_{\mathrm{i}}=k_{\mathrm{i}} a, \quad m_{\mathrm{a}}=k_{\mathrm{a}} a, \quad k=\omega / r .
\end{gathered}
$$

Ferner ist $m_{\mathrm{a}}^{2}-m_{\mathrm{i}}^{2}=\left(\omega^{2} a^{2} / c^{2}\right)\left(\varepsilon_{\mathrm{a}}-\varepsilon_{\mathrm{i}}\right), \quad$ und da $\varepsilon_{\mathrm{a}}=1-\left(\omega_{0}^{2} / \omega^{2}\right)$ ist,

$$
\left(m_{\mathrm{i}}^{2} / \varepsilon_{\mathrm{i}}\right)-m_{\mathrm{a}}^{2}=\left(a^{2} / c^{2}\right) \omega_{0}^{2},
$$

1 F. B or g n is, Ann. Physik (5) 35, 375 [1939]. 
oder, wenn für $\omega<\omega_{0} \quad m_{\mathrm{a}}$ imaginär wird, $m_{\mathbf{a}}=-j m_{\mathbf{a}}{ }^{\prime}$,

$$
\left(m_{\mathrm{i}}^{2} / \varepsilon_{\mathrm{i}}\right)+m_{\mathrm{a}}^{\prime 2}=\left(a^{2} / c^{2}\right) \omega_{0}^{2} .
$$

Mit $l=1$ und Einführung der Werte der Besselschen Funktion halbzahliger Ordnung wird aus (8)

$$
\frac{1}{m_{\mathrm{i}}^{2}}-\frac{\operatorname{tg} m_{\mathrm{i}}}{\operatorname{tg} m_{\mathrm{i}}-m_{\mathrm{i}}}=-\left(\frac{1}{m_{\mathrm{a}}^{\prime 2}}+\frac{1}{1+m_{\mathrm{a}}^{\prime}}\right) .
$$

Abb. 1 zeigt den Zusammenhang von $m_{\mathrm{i}}$ und $m_{\mathrm{a}}{ }^{\prime}$ nach Gl. (10). Diese Kurven sind zu schneiden mit den Ellipsen der Gl. (9), um im Punkt $\mathrm{P}$ die Werte $m_{\mathrm{a}}{ }^{\prime}$ und $m_{\mathrm{i}}$ festzulegen.

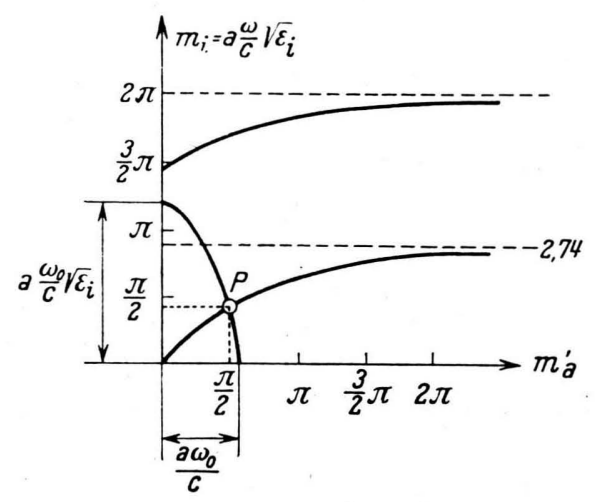

Abb. 1. Zusammenhang von $m_{\mathrm{i}}$ und $m_{\mathrm{a}}{ }^{\prime}$ nach Gl. (10).

Aus $m_{\mathrm{i}}$ folgt die Eigenfrequenz mit $\omega=m_{\mathrm{i}} \frac{c}{a} \frac{1}{\sqrt{\varepsilon_{\mathrm{i}}}}$.

Man sieht zunächst, daß ungedämpfte Schwingungen mit imaginärem $m_{\mathrm{a}}$, also ohne Ausstrahlung, möglich sind. Aus der Abb. 1 sieht man, daß $\omega$ immer kleiner als $\omega_{0}$ ist, die D.K. $\varepsilon_{\mathrm{P}}$ des Plasmas aber negativ ist. Je kleiner $\omega_{0}$ ist, desto kleiner wird auch $\omega$. Für kleine $m_{\mathrm{a}}{ }^{\prime}$ und $m_{\mathrm{i}}$ ist $m_{\mathrm{i}}=\sqrt{2} m_{\mathrm{a}}{ }^{\prime}$, $\omega_{\mathrm{ei}}^{2}=\omega_{0}^{2} \frac{1}{1+\left(\varepsilon_{\mathrm{i}} / 2\right)}$.

Mit $\omega_{0}$ verschwindet auch $\omega$, d. h. ohne bewegte Elektronen ist dieser Schwingungstyp unmöglich. Da

$$
\begin{aligned}
& H_{1 / 2}^{2}(-j x)=\frac{1}{\sqrt{j}} \sqrt{\frac{2}{\pi x}} \exp (-x) \text { und } \\
& H_{3 / 2}^{2}(-j x)=\sqrt{j} \sqrt{\frac{2}{\pi x}}\left(1+\frac{1}{x}\right) \exp (-x)
\end{aligned}
$$

uńd $x=k_{\mathrm{a}}{ }^{\prime} r=m_{\mathrm{a}}{ }^{\prime}(r / a)$ ist, bedeutet kleines $m_{\mathrm{a}}{ }^{\prime}$ einen sehr langsamen exponentiellen Feldabfall nach außen. Es ist also bei kleinem $\omega_{0} / c$ ein relativ großes räumliches Plasmavolumen, das sich an den Schwingungen beteiligt. Wächst $\omega_{0} / c$, so wachsen auch $m_{\mathrm{i}}$ und $m_{\mathrm{a}}{ }^{\prime} . m_{\mathrm{a}}{ }^{\prime}$ kann beliebig groß werden, d. h. das Feld fällt immer rascher exponentiell nach außen ab und verteilt sich bei großem $m_{\mathrm{a}}{ }^{\prime}$ nur auf' eine dünne Schicht um die dielektrische Kugel. Dagegen kann $m_{\mathrm{i}}$ höchstens bis zum Wert 2,74 anwachsen, der der Eigenfrequenz der dielektrischen Kugel mit Metallumhüllung entspricht (s. Abschn. Ia). Es ist dies die höchstmögliche Frequenz der untersten Kurve der Abb. 1. Bei größerem $a \omega_{0} / c$ treten dagegen auch Schnitte mit den höher liegenden $m_{\mathrm{i}}-m_{\mathrm{a}}$ '-

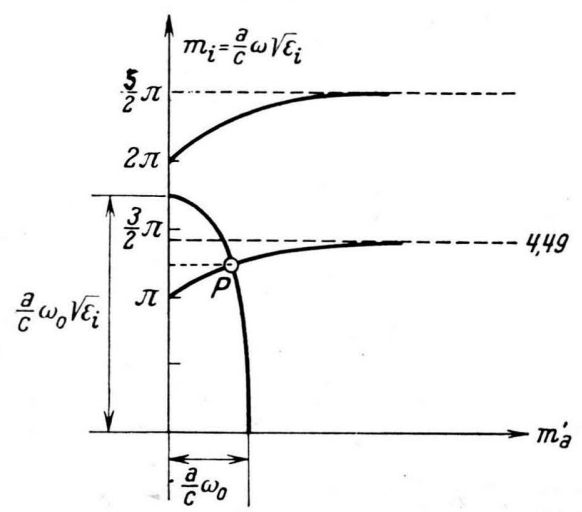

Abb. 2. Zusammenhang von $m_{\mathrm{i}}$ und $m_{\mathbf{a}}{ }^{\prime}$ nach Gl. (12).

Kurven auf, die höheren Frequenzen (Oberwellen) dieses Typs entsprechen.

b) Für die transviersale $E-W e l l e$ lautet die Stetigkeitsbedingung für $r=a$

$$
\frac{m_{\mathrm{i}} Z_{l-1 / 2}^{i}\left(m_{\mathrm{i}}\right)}{Z_{l+1 / 2}^{i}\left(m_{\mathrm{i}}\right)}=\frac{m_{\mathrm{a}} Z_{l-1 / 2}^{a}\left(m_{\mathrm{a}}\right)}{Z_{l+1 / 2}^{a}\left(m_{\mathrm{a}}\right)},
$$

oder (für $l=1$ )

$$
\frac{m_{\mathrm{i}} \operatorname{tg} m_{\mathrm{i}}}{-1+\frac{1}{m_{\mathrm{i}}} \operatorname{tg} m_{\mathrm{i}}}=-\frac{m_{\mathrm{a}}^{\prime 2}}{m_{\mathrm{a}}^{\prime}+1} .
$$

Abb. 2 zeigt den Zusammenhang von $m_{\mathrm{i}}$ und $m_{\mathbf{a}}$ nach Gl. (12).

Die Kurven sind wieder mit der Ellipse mit den Halbachsen $(a / c) \omega_{0} V \varepsilon_{\mathrm{i}}$ und $(a / c) \omega_{0}$ zu schneiden, um den Betriebspunkt $\mathrm{P}$ zu bekommen. Hier gibt es einen unteren kritischen Wert der Plasmadichte, $(a / c) \omega_{0} \sqrt{\varepsilon_{\mathbf{i}}}=\pi$, oberhalb dessen überhaupt erst Schwingungen möglich sind. Es ist allgemein $\omega<\omega_{0}$; nur in diesem kritischen Punkt 
wird $\omega=\omega_{0}$. Je größer der Kugelradius und ihre D.K. ist, um so tiefer liegt die kritische Frequenz. Mit wachsender Plasmadichte wächst auch $\omega_{\text {ei }}$ und $m_{\mathrm{a}}{ }^{\prime}$. Auf der untersten Kurve von Abb. 2 kann $m_{\mathrm{i}}$ nicht über 4,49 wachsen bei $m_{\mathrm{a}}{ }^{\prime} \rightarrow \infty$. Diesem Werte entspricht die Bedingung $\operatorname{tg} m_{\mathrm{i}}=m_{\mathrm{i}}$, d. h. der leitend begrenzten Hohlkugel (s. Abschn. Ib). Der kleinste Wert, den $m_{\mathrm{a}}^{\prime}$ annehmen kann, ist $\pi / \sqrt{\varepsilon_{\mathrm{i}}}$, d. h. der felderfüllte Raum im Plasma ist.schon bei der unteren Grenzfrequenz beschränkt und schrumpft um so mehr zusammen, je größer $(a / c) \omega_{0}$ ist.

\section{Gut leitende $\mathrm{Kugel}$}

- in einer Plasmaatmosphäre

Dieser Fall ist als Grenzfall für $\varepsilon_{\mathbf{i}} \rightarrow \infty$ aus Gl. (8) und bzw. (11) abzuleiten und ergibt für die transversale $H$-Welle:

$$
l=m_{\mathrm{a}} \frac{H_{1 / 2}^{2}\left(m_{\mathrm{a}}\right)}{H_{3 / 2}^{2}\left(m_{\mathrm{a}}\right)},
$$

aus der für $m_{\mathrm{a}}=\frac{j}{2} \pm \frac{1}{2} \sqrt{3}$ folgt. Es folgt dies auch aus der Bedingung $E_{\Theta}=0$ für $r=a$ nach Gl. (4). Mit $m_{\mathrm{a}}=a \frac{\omega}{c} \sqrt{\varepsilon_{\mathrm{P}}}$ folgt für $\omega$

$$
\omega^{2}=\omega_{0}^{2}+\left(c^{2} / a^{2}\right)\left(\frac{1}{2} \pm i \frac{V 3}{2}\right),
$$

und mit $\omega=\omega_{\mathrm{ei}}+j \beta$

$2 \omega_{\mathrm{ei}}^{2}=\left(\omega_{0}^{2}+\frac{c^{2}}{2 a^{2}}\right)\left[1+\sqrt{1+\frac{3}{4}} \frac{c^{4}}{a^{4}} \frac{1}{\left(\omega_{0}^{2}+c^{2} / 2 a^{2}\right)^{2}}\right]$,

$2 \beta^{2}=\left(\omega_{0}^{2}+\frac{\mathrm{c}^{2}}{2 a^{2}}\right)\left[-1+\sqrt{1+\frac{3}{4} \frac{c^{4}}{a^{4}} \frac{1}{\left(\omega_{0}^{2}+c^{2} / 2 a^{2}\right)^{2}}}\right]$.

Die Frequenz ist komplex mit positiv imaginärem Teil, die Schwingung ist gedämpft. Die Eigen- ${ }^{\circ}$ frequenz liegt über der Plasmaeigenfrequenz $\omega_{0}$, $\varepsilon_{\mathrm{P}}$ ist positiv.

Wir wollen zwei Grenzfälle betrachten:

1. $\omega_{0}^{2} \ll c^{2} / 2 a^{2}, \quad \omega_{\mathrm{ei}}^{2} \approx \frac{3}{4} c^{2} / a^{2} \gg \omega_{0}^{2}, \quad \beta^{2}=c^{2} / 4 a^{2}$.

Die Kugel schwingt in einem sehr dünnen Plasma, als ob sie sich in Luft befände. Das gilt, wenn $a^{2} \ll c^{2} / 2 \omega_{0}{ }^{2}$.

2. $\omega_{0}^{2} \gg c^{2} / a^{2}, \omega_{\mathrm{ei}}^{2} \approx \omega_{0}^{2} \gg c^{2} / 2 a^{2}, \beta=\frac{c}{2 a} \sqrt{3}\left(\frac{c / 2 a}{\omega_{0}}\right)$. $c / 2 a$ ist die Dämpfung für $\varepsilon_{r}=1$. Die Kugel schwingt mit der Eigenfrequenz des Plasmas, die viel größer ist als ihre Eigenfrequenz in Luft. Die Dämpfung ist aber viel kleiner, als sie in Luft wäre. Sie ist je Periode $2 \pi \beta / \omega_{\mathrm{ei}}=2 \pi \sqrt{3}\left(\frac{\mathrm{c} / 2 a}{\omega_{0}}\right)^{2}$ und kann sehr klein werden. Nimmt man als Beispiel $\omega_{0}=2 \pi 10^{9} \mathrm{sec}^{-1}$, was etwa einem mittleren Plasma in einer Niederdruck-Hg-Entladung entspricht, so ist für $a \gg 2,5 \mathrm{~cm}$ die obige Bedingung erfüllt. Oder nimmt man als sehr große Kugel die Erde mit $a=6000 \mathrm{~km}$ an, so ist deren Eigenfrequenz in Luft $\omega_{\mathrm{ei}_{\varepsilon_{r}}=1}=25 \sqrt{3} \mathrm{sec}^{-1}$, und es müßte $\omega_{0} \gg 25 \mathrm{sec}^{-1}$ sein, damit die obige Bedingung erfüllt ist. Mit $\omega_{0} \approx 10^{6} \sec ^{-1}$ z. B. wäre dann $\beta=10^{-3} \mathrm{sec}^{-1}$. In einer derartigen weit ausgedehnten Atmosphäre wäre die Eigenfrequenz $f_{\mathrm{ei}}=1,5 \cdot 10^{5} \mathrm{H}$ und die Schwingung nach $10^{3} \mathrm{sec}$ auf $1 / e$ gefallen.

Für die transversale $E$-Welle wäre analog nach den Gln. (11) und (5) $Z_{3 / 2}\left(m_{\mathrm{a}}\right)=0 \mathrm{zu}$ setzen, d.h. $m_{\mathrm{a}}=j$, woraus $\omega_{\mathrm{ei}}=\sqrt{\omega_{0}{ }^{2}-c^{2} a^{2}}$ folgt.

Im Gegensatz zum vorigen Fall wäre hier für $\omega_{0}>a_{l}^{\prime} c$ eine periodische ungedämpfte Schwingung möglich, die aber physikalisch nicht realisierbar ist, da die Felder nach außen exponentiell zunehmen müßten. Für $\omega_{0}<c / a$ wird $\omega_{\mathrm{ei}}$ rein imaginär, die Felder klingen ohne Schwingungen ab. Die Wurzeln von $m_{\mathrm{a}}$ für die Oberschwingungen in beiden Fällen können der Arbeit von $\mathrm{P}$. D e b y e ${ }^{2}$ entnommen werden.

\section{Plasmakugel, von Luft umgeben}

Wie bekannt, ergeben dielektrische Kugeln im allgemeinen komplexe Lösungen der Stetigkeitsbedingungen, d. h. gedämpfte Eigenschwingungen (s. z. B. Debye ${ }^{2}$ ). Wir suchen hier nur die ungedämpften bzw. nahezu ungedämpften Eigenschwingungen, auf die Deby e ${ }^{2}$ hinweist. Sie treten nur dann auf, wenn das Argument $m_{\mathrm{a}}$ der Hankelschen Funktion im Außenraum sehr klein wird. Wir suchen sie speziell für imaginäres $m_{\mathrm{i}}$ zu bestimmen $\left(\varepsilon_{\mathrm{P}}\right.$ negativ, $\left.\omega<\omega_{0}\right)$, d. h. die niedersten Frequenzen, die bei Anwesenheit eines Plasmas auftreten können.

Führt man wieder die Werte der halbzahligen Bessel- und Hankel-Funktionen ein, so entsteht
2 P. Debye, Ann. Physik
(4) 30, 75 [1909]. 


$$
\begin{aligned}
& \frac{1}{m_{\mathrm{i}}^{2}}\left[1-\frac{m_{\mathrm{i}} \operatorname{tg} m_{\mathrm{i}}}{-1+\left(\operatorname{tg} m_{\mathrm{i}} / m_{\mathrm{i}}\right)}\right] \\
& =\frac{1}{m_{\mathrm{a}}^{2}}\left[1-j \frac{m_{\mathrm{a}}}{-1+\left(j / m_{\mathrm{a}}\right)}\right] .
\end{aligned}
$$

und mit $m_{\mathbf{i}}=j m_{\mathbf{i}}{ }^{\prime}$

$$
\begin{gathered}
-\frac{1}{m_{\mathrm{i}}^{\prime 2}}\left[1+\frac{m_{\mathrm{i}}^{\prime} \mathfrak{I g} m_{\mathrm{i}}^{\prime}}{-1+\left(\mathfrak{T g} m_{\mathrm{i}}^{\prime} / m_{\mathrm{i}}^{\prime}\right)}\right] \\
=\frac{1}{m_{\mathrm{a}}^{2}}\left[1-\frac{m_{\mathrm{a}}^{2}}{1+j m_{\mathrm{a}}}\right] .
\end{gathered}
$$

Wird $m_{\mathrm{a}}$ sehr klein, so strebt die rechte Seite gegen $\left(1 / m_{\mathbf{a}}{ }^{2}\right)\left(1-m_{\mathbf{a}}{ }^{2}\right)$, sie wird also sehr groß und reell. Für die linke Seite von (13) muß dann $\operatorname{tg} m_{\mathrm{i}}=m_{\mathrm{i}}$ werden, was zu unendlich vielen Wurzeln führt, wie sie Debye ${ }^{2}$ für positives $\varepsilon_{\mathbf{i}}$ bestimmt hat: Für imaginäres $m_{i}$ muß $m_{i}{ }^{\prime}$ auch sehr klein werden, damit die linke Seite von (14) auch sehr groß wird. Sie strebt dann gegen $\left(-1 / m_{i}{ }^{2}\right)$ $\left(-2+m_{\mathrm{i}}{ }^{2}\right)$. Die Lösung der Gl. (14) ist dann in erster Näherung $m_{\mathrm{i}}{ }^{2}=2 m_{\mathrm{a}}{ }^{2}$, so daß $m_{\mathrm{a}}{ }^{2}+m_{\mathrm{i}}{ }^{2}$ $\approx 3 m_{\mathrm{a}}{ }^{2}=\omega_{0}{ }^{2} a^{2} / c^{2}$ wird, d. h. $m_{\mathrm{d}}=(\omega / c) \mathrm{a}$ $\approx\left(\omega_{0} / \sqrt{3}\right)(a / c)$ und die Eigenfrequenz $\omega_{\mathrm{ei}}$ $\approx \omega_{0} / \sqrt{3}$. Die genauere Rechnung ergibt komplexe Werte von $m_{\mathbf{i}}$ und $m_{\mathbf{a}}$, aber das Dämpfungsglied ist klein von der 3. Ordnung. Es sind demnach ungedämpfte (bzw. sehr schwach gedämpfte) Schwingungen mit $\omega<\omega_{0}, \varepsilon_{\mathrm{P}}<0$ möglich, wenn $\left(\omega_{0} / V \overline{3}\right)(a / c) \ll 1$ ist. Da die tiefste Eigenfrequenz einer Metallkugel in Luft $\omega_{\mathrm{ei}_{\mathrm{L}}}=(c / a)$ $(\sqrt{3} / 2)$ ist, bedeutet dies $\omega_{0} \ll 2 \omega_{\text {ci }}$.

Der Kugelradius spielt bei der Frequenz $\omega_{\text {ei }}$ keine Rolle. Je größer der Kugelradius ist, desto geringer ist die zulässige Plasmadichte. Für die Wellenlänge $\lambda_{0}$, die der Plasmaeigenfrequenz $\omega_{0}$ entspricht, gilt $\lambda_{0} \gg(2 \pi / \sqrt{3}) a=3,6 a ; z$. B. fuir $a=100 \mathrm{~cm} \mathrm{muß} \lambda_{0} \gg 3,6 \mathrm{~m} \mathrm{sein,} \mathrm{für} a=1 \mathrm{~cm}$. $\lambda_{0} \gg 3,6 \mathrm{~cm}$.

Für die Verteilung der Felder gelten die Gln. (4). Mit

$$
k_{\mathrm{i}} r=\xi_{\mathrm{i}}=j \xi_{\mathrm{i}}^{\prime}, \quad m_{\mathrm{i}}^{\prime}=j m_{\mathrm{i}}=k_{\mathrm{i}}^{\prime} a
$$

ergibt die Darstellung der Bessel-Funktionen $J_{1 / 2}$ und $J_{3 / 2}$ für kleines imaginäres Argument:

$$
B_{\Upsilon_{\mathrm{i}}}=-\frac{1}{3} \sqrt{\frac{2}{\pi}} k_{\mathrm{i}}^{\prime} \xi_{\mathrm{i}}^{\prime} \sin \Theta C_{\mathrm{i}},
$$

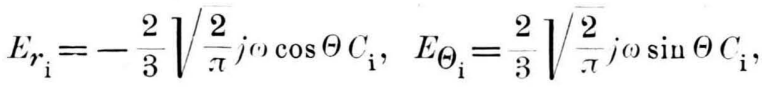

so daß die elektrischen und magnetischen Felder praktisch um $90^{\circ}$ in der Phase gegeneinander verschoben sind. Das Magnetfeld wächst nach: außen proportional mit $r$ und besteht aus Kreisen um die $z$-Achse. Das elektrische Feld ist homogen und iiberall parallel zur $z$-Achse, $E_{\Theta} / E_{r}=-\operatorname{tg}(-)$. Seine Größe ist $-j \omega \frac{2}{3} \sqrt{\frac{2}{\pi}} C_{i}$.

Im Außenraum ergeben sich die Felder aus den Hankel-Funktionen $H_{1 / 2}^{2}$ und $H_{3 / 2}^{2}$ mit dem Argument $\xi_{\mathrm{a}}=k_{\mathrm{a}} r, \quad m_{\mathrm{a}}=h_{\mathrm{a}} a \mathrm{zu}$

$$
B_{T_{\mathrm{a}}}=C_{\mathrm{a}} k_{\mathrm{a}} \sqrt{\frac{2}{T}} \frac{1}{\xi_{\mathrm{a}}^{2}}\left(-\xi_{\mathrm{a}}+j\right) \exp (-j \xi u) \sin \Theta ;
$$

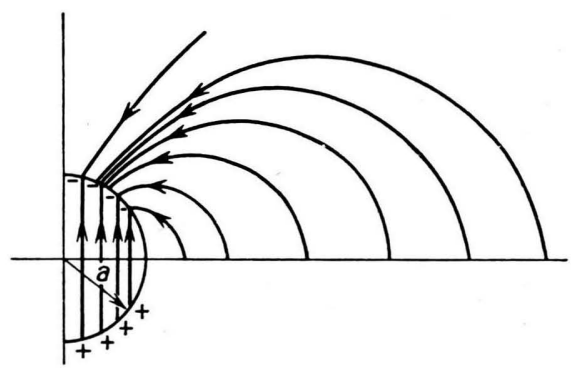

Abl. 3. Skizze der Feldverteilung innerhalb und außerhalb der Kugel.

$$
E_{r_{\mathrm{a}}}=-C_{\mathrm{a}} 2 j \omega \sqrt{\frac{2}{\pi} \frac{1}{\xi_{\mathrm{a}}^{3}}}\left(j-\xi_{\mathrm{a}}\right) \exp (-j \xi a) \cos \Theta
$$

$E_{\Theta_{\mathrm{a}}}=-C_{\mathrm{a}} j \omega \sqrt{\frac{2}{\pi}} \frac{1}{\xi_{\mathrm{a}}^{3}}\left[-\xi_{\mathrm{a}}+j\left(1-\xi_{\mathrm{a}}^{2}\right)\right] \exp (-j \xi a) \sin \Theta$

In der Nähe der Kugeloberfläche, wo $\xi_{\mathrm{a}}$ sehr klein ist, gilt

$$
\begin{aligned}
& B_{T_{\mathrm{a}}}=C_{\mathrm{a}} k_{\mathrm{a}} \sqrt{\frac{2}{\top} \frac{j}{\xi_{\mathrm{a}}^{2}}} \exp (-j \xi a) \sin \Theta ; \\
& E_{r_{\mathrm{a}}}=C_{\mathrm{a}} 2 \omega \sqrt{\frac{2}{\pi}} \frac{1}{\xi_{\mathrm{a}}^{3}} \exp (-j \xi a) \cos \Theta ; \\
& E_{\Theta_{\mathrm{a}}}=C_{\mathrm{a}} \Theta \sqrt{\frac{2}{\tau}} \frac{1}{\xi_{\mathrm{a}}^{3}} \exp (-j \xi a) \sin \Theta,
\end{aligned}
$$

so daß hier elektrisches und magnetisches Feld auch nahezu um $90^{\circ}$ in der Phase gegeneinander verschoben sind. Nach außen fallen die Felder rasch mit $1 / \xi_{\mathrm{a}}{ }^{2}$ bzw. $1 / \xi_{\mathrm{a}} 3 \mathrm{ab}$.

Aus

$$
E_{\Theta} / E_{r}=1 / 2 \operatorname{tg} \Theta=r d \Theta d r
$$

folgt die Kraftliniengleichıng in der $r, \Theta$-Ebene zul $r=h \sin ^{2} \Theta$. 
Abb. 3 gibt eine Skizze der Feldverteilung innerhalb und außerhalb der Kugel. Da die D.K. des Plasmas negativ ist, sind die Normalkomponenten von $\tilde{夭}$ an der Kugeloberfläche einander entgegengesetzt gerichtet, und es treten an der Kugeloberfläche infolge der bewegten Elektronen freie positive und negative Ladungen auf.

Aus der Grenzbedingung an der Oberfläche ergibt sich das Verhältnis der Konstanten $C_{\mathrm{a}}$ und $C_{\mathrm{i}}$ für kleines $m_{\mathrm{a}} \mathrm{zu}$

$$
C_{\mathrm{a}} / C_{\mathrm{i}}=j 2 / 3 m_{\mathrm{a}}^{3} \exp (j m a) \text {. }
$$

\section{Strahlung der Kugel}

Die Gesàmtstrahlung ist gegeben durch

$\frac{1}{2} \int_{0}^{\pi} \frac{E_{\Theta} B_{\varphi}^{+}}{\mu_{\mathrm{u}}} r^{2} \sin \Theta d \Theta=-\frac{c}{\mu_{0}} \frac{2}{3} \frac{2}{\pi} \frac{1}{\xi_{\mathrm{a}}^{3}}\left(j-\xi_{\mathrm{a}}^{3}\right) C_{\mathrm{a}}{ }^{2}$.

Wird dies auf den gesamten Strom bezogen, der die Kugel in der Aquatorialebene durchfließt,

$$
I=\oint_{\substack{\Theta=90^{\circ} \\ r=a}} H_{s} d s=\frac{1}{\mu_{0}} B_{\substack{\Theta=90^{\circ} \\ r=a}} 2 \pi a=\frac{4 \pi}{3} \sqrt{\frac{2}{\pi}} \frac{m_{\mathrm{a}}^{2}}{\mu_{0}} C_{\mathrm{i}},
$$

da $m_{\mathrm{i}}{ }^{2}=2 m_{\mathrm{a}}{ }^{2}$ für kleine $m_{\mathrm{i}}$ und $m_{\mathrm{a}}$, so folgt mit

$$
\begin{aligned}
& \frac{\left|C_{\mathrm{a}}\right|^{2}}{\mid C_{\mathrm{i}}{ }^{2}}=\frac{4}{9} m_{\mathrm{a}}{ }^{6}[\text { Gl. (15) }] \\
& \qquad \mid C_{\mathrm{a}}{ }^{2}=\frac{1}{8 \pi} m_{\mathrm{a}}^{2} I^{2} \mu_{0}^{2} .
\end{aligned}
$$

Dadurch ist die mittlere gestrahlte Leistung

$$
\begin{array}{r}
N=I^{2} \frac{1}{6 \pi^{2}} \sqrt{\frac{\mu_{0}}{\varepsilon_{0}}} m_{\mathrm{a}}^{2} \frac{1}{\xi_{\mathrm{a}}^{3}}\left(\xi_{\mathrm{a}}^{3}-j\right), \\
\left(V !_{0} / \varepsilon_{0}=120 \pi \mathrm{Ohm}\right) .
\end{array}
$$

Wenn man sie vergleicht mit der Strahlung eines Dipols vom Strom $J$ und der Länge $l$

$$
N_{\mathrm{D}}=I^{2} \frac{1}{3} \pi \sqrt{\frac{\mu_{0}}{\varepsilon_{0}}} \frac{l^{2}}{\lambda^{2}}, \quad(\hat{\lambda}=\text { Vakuumwellenlänge })
$$

so ist bei Gleichheit der Strahlungen bei demselben Strom

$$
l / \lambda=\left(1 / \sqrt{2 \pi^{3}}\right) m_{\mathrm{a}} \text {, d.h. } l \ll \lambda, \text { wenn } m_{\mathrm{a}} \ll 1 .
$$

Bei genügend kleinem $m_{\mathrm{a}}$ wird die abgestrahlte Leistung tatsächlich sehr gering, so daß die Schwingung nur sehr wenig gedämpft ist.

Der 2. radiale transuersale E-Wellent y p läßt einen Schwingungstyp dieser Art nicht zu, da die Stetigkeitsbedingung keine Lösung für kleine $m_{\mathrm{a}}$ zuläßt.

\title{
Durch Dehnung bewirkter Vorzeichenwechsel der Magnetostriktion
}

\author{
Von Wilhelm Braunewell und Eckart Vogt \\ Aus dem Physikalischen Institut der Universität Marburg \\ (Z. Naturforschg. 4 a, 491-495 [1949]; eingegangen am 20. März 1949) \\ Walther Gerlach zu seinem 60. Geburtstag.
}

\begin{abstract}
Während der als Villari-Umkehr bekannte Vorzeichenwechsel der Magnetostriktion des Eisens von der Aufeinanderfolge von 90-Wandverschiebungen und Drehprozessen bei fortschreitender Magnetisierung herrührt, findet man bei einer Heusler-Legierung und bei Fe-Ni-Legierungen eine andere Art des Vorzeichenwechsels. Sie läßt sich darauf zurückführen, daß entweder Zugspannung eine Änderung der spontanen Gitterverzerrung bewirkt oder daß durch Zug die Magnetisierungsvektoren unter Umständen trotz positiver Magnetostriktion aus der Zugrichtung herausgedreht werden können.
\end{abstract}

1.

$\mathrm{E}$ in Vorzeichenwechsel der Magnetostriktion (MS) ist wohl bekannt und in seinem $\mathrm{Zu}^{-}$ standekommen überzeugend gedeutet beim Eisen. Im wachsenden Magnetfeld erfährt ein entmagnetisierter Weicheisenstab zuerst eine Verlängerung, die dann wieder zurückgeht und schließlich in eine Verkürzung ausläuft (Abb. 1). Elastische
Dehnung (Zugspannung $\sigma$ ) verringert die anfängliche Verlängerung; sie wird schließlich durch eine kritische Mindest-Zugspannung $\left(\sigma_{k}\right)$ überhaupt unterdrückt. Das Wertepaar $(H ; \sigma)$ des Maximums in Abb.1, bei der die Verlängerung in Verkürzung übergeht, nennt man den Villari-Punkt nach E. Vill a r i, der schon 1865 die Erscheinung entdeckte durch seine Beobach- 